\title{
Right Versus Left-Sided Colon Cancer: Analysis of Epidemiology in Lebanese Patients
}

\author{
Ahmad S Khalil ${ }^{1}$, Kamal H Al Zahran ${ }^{2 *}$, Evelyne G Helou ${ }^{1}$ and Antoine A Abboud ${ }^{1}$ \\ ${ }^{1}$ Hematology Oncology department, Gastroenterology department, Geitawi hospital university medical center, Ashrafieh, Beirut \\ ${ }^{2}$ Hematology Oncology department, Middle East Institute of Health (MEIH), university hospital, Bsalim, Mount Lebanon, Lebanon
}

*Corresponding author: Kamal H Al Zahran, Hematology Oncology department, Middle East Institute of Health (MEIH), university hospital, Bsalim, Mount Lebanon, Lebanon

\begin{abstract}
Objective: Recent studies have showed that right-sided colon cancer was more aggressive than left-sided and was more common in female and older patients. We examined the incidence of colon cancer by location (right versus left sided) in one university medical center, located in Beirut, Lebanon.

Methods: We collected the data of patients with colon cancer diagnosed between the years of 2011 and 2016 in Geitawi University Hospital, comparing the epidemiology of cases with right versus left sided colon cancer; concerning the following variables: age, sex, RAS status and the stage according to the American Joint Commission of Cancer AJCC.

Results: 96 patients were included, $61 \%$ had left-sided, 36\% had right-sided, 1.5\% had cancer in the transverse colon and $1.5 \%$ had synchronous left and right sided colon cancer. Patients with transverse and both right and left sided colon cancer were excluded from the study. We examined the incidence in patients under the age of 50; in whom screening is not recommended, versus patients over the age of 50; in whom screening is recommended. Patients with left-sided colon cancer were more common, more likely to be younger, to be males and to be diagnosed with a more advanced stage. In patients under the age of 50, 20\% had cancer in the right and $80 \%$ had left-sided colon cancer.
\end{abstract}

Conclusion: Patients with right-sided were less common, predominantly males, older and with less aggressive stage than patients with left-sided colon cancer in a sub-group of Lebanese population.

Keywords: Colon Cancer; Right Sided; Left Sided; Epidemiology

\section{Introduction}

Colorectal cancer is one of the most commonly diagnosed cancers worldwide with over 1.2 million new cases and 608,700 deaths estimated to occur annually [1]. Colorectal cancer (CRC) is the second leading cause ofcancer-related deaths for both men and women in the United States, with 147,000 new occurrences and 50,000 deaths in 2009 [2]. Over the past years, the distinction between right-sided and left-sided colon cancer has been brought into consideration, regarding epidemiology, clinical presentation, pathology, and genetic mutations [3] Bufill in 1990 was the first to propose this, knowing that there are a number of differences between the sides of the bowel, for example the embryological beginnings; with the right bowel arising from the midgut and the left side from the hindgut, in addition to the difference in the vascular supply of each side [4].Epidemiological studies have demonstrated a gender and age relationship with a higher incidence of right colon cancer (RCC) in women and elderly people. Therefore, it has been suggested to consider colo-rectal cancer as three distinct tumor entities: right colon cancer (RCC), left colon cancer (LCC) and rectal cancer [5]. Right-sided colon cancers tend to be bulky, exophytic, polypoid lesions growing into the colon lumen and causing anemia. However, left-sided colon cancers tend to be infiltrating, constricting lesions en-circling the colorectal lumen and causing obstruction [6]. The idea of personalized medicine was introduced to the treatment of metastatic colorectal cancer (mCRC) when KRAS codon $12 / 13$ mutations were identified as negative predictors of anti-EGFR-antibody (EGFR-mAB) treatment [7]. While there are 
many publications worldwide regarding this difference, it's the first time in Lebanon we approach this issue. We studied patients with colon cancer admitted to Geitawi University Medical Center in an interval of 6 years (Figure 1).

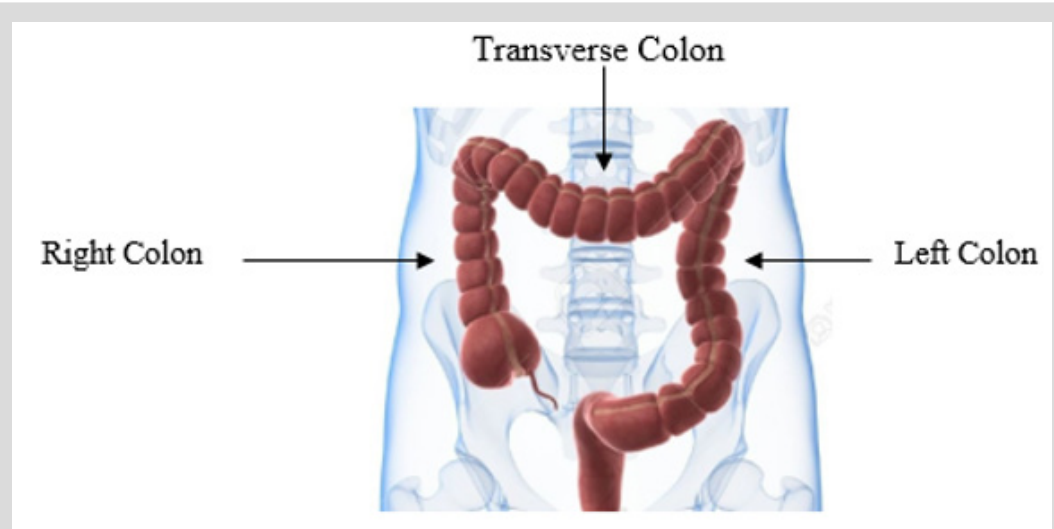

Figure 1: The different entity of colon cancer Right Colon (ascending colon + hepatic flexure) Transverse Colon Left Colon (splenic flexure + descending colon + sigmoid).

\section{Methods}

\section{Data selection}

The data was detected from the archive of data base in Geitawi University Medical Center in Beirut. Patients selected were those diagnosed with colon cancer between the year of 2011 and 2016. The data selected included: patients age, tumor location, tumor stage and RAS status in stage 4 colon cancer.

\section{Patient selection}

All patients who were diagnosed with primary adenocarcinoma of the colon in Geitawi hospital from 2011 to 2016 were included in the study, 96 patients were identified, 22 patients were excluded either because the location of the cancer was not exactly identified or because the workup and management was continued outside the hospital, also 2 patients were excluded because the Cancer was located in the transverse or synchronous side right and left colon. Inclusion criteria were as follows:

a) All patients diagnosed with colon cancer between 2011 and 2016

Exclusion criteria were as follows:

b) Transverse colon cancer

c) Synchronous left and right sided colon cancer

d) Patients continued the care out of the hospital

e) Patient without exactly primary location of colon cancer

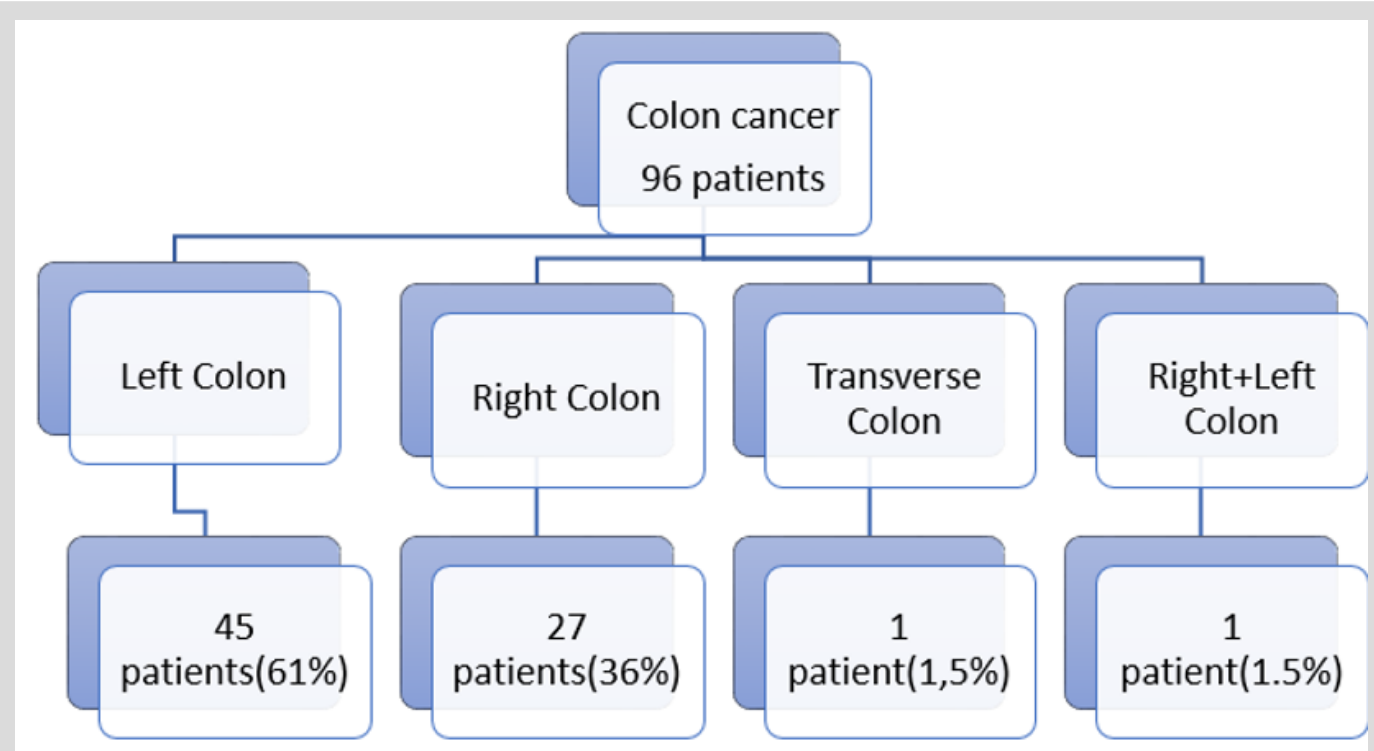

Figure 2: Data collection and sidedness of colon cancer.

After considering the above criteria, the patient pool consisted of 72 patients. They were distributed into two groups according to the colonic tumor site: 45 cases were found to be LCC and 27 were
RCC. We collected the information considering the age, sex, stage according to AJCC American Joint Committee on Cancer and RAS status because Ras mutation was identified as a negative predictor 
of anti-EGFR-antibody (EGFR-mAB) treatment in each group. Then we divided the patients according to the age into two groups, one included patients $<50$ years of age and the other included patients $\geq$ 50 years (Figure 2).

\section{Result}

Out of the 72 patients, two thirds $62.5 \%$ had LCC, $37.7 \%$ were right-sided, $57 \%$ were males, $43.5 \%$ were females, and the mean age of the patients upon diagnoses was 65 years (Table1). In the Left-sided subgroup, the mean age was 62.5 years, $53 \%$ were males, $47 \%$ were females, and according to the AJCC staging system; $49 \%$ had tumor with stage 4 upon presentation and 51\% had tumor with stage less than 4.The RAS status was found to be wild in $30 \%$ and mutated in $61 \%$ of cases, knowing that the mutation was not done in $9 \%$ of patients.In the right-sided subgroup, the mean age was 68 years, $63 \%$ were males and $37 \%$ were females. Moreover, according to the AJCC staging system, $37 \%$ had stage 4 tumor upon presentation and $63 \%$ had tumor with stage less than 4 . The RAS was wild in $20 \%$ and mutated in $30 \%$ of patients. The mutation was not tested in $50 \%$ of patients, of whom $75 \%$ did not test because they decided to receive palliative treatment only. With respect to the two groups divided according to age $<50$ and $\geq 50$ years (Table 2), $13.9 \%$ of the total number of patients were under the age of 50 . In the latter subgroup, $80 \%$ had LCC, $40 \%$ were males, $60 \%$ were females, and the mean age was 41 years. Moreover $40 \%$ were with stage 4 tumor and $60 \%$ had tumor with stage $<4$ upon diagnosis. Regarding the subgroup including patients $\geq 50$ years, 59.7\% LCC, $59.7 \%$ were males and $40.3 \%$ were females. The mean age was 69 years, $46.8 \%$ were with stage 4 tumor and $53.2 \%$ had tumor in stage $<4$ upon diagnosis (Figure 3) (Tables 1-2).



Figure 3: Epidemiologic differences between the 2-age group.

Table 1: Epidemiologic differences between right-sided and left-sided colon cancer.

\begin{tabular}{|c|c|c|c|}
\hline & & Left Colon & Right Colon \\
\hline Male & & $24(53 \%)$ & $17(63 \%)$ \\
\hline Female & & $21(47 \%)$ & $10(37 \%)$ \\
\hline Mean age & & 62.5 & 68.8 \\
\hline \multirow{2}{*}{ Age $<50$ years } & Mean age & 42 & 45 \\
\cline { 2 - 4 } & Number & $8(18 \%)$ & $2(7.4 \%)$ \\
\hline \multirow{2}{*}{ Age $\geq 50$ years } & Mean age & 67 & 71 \\
\cline { 2 - 4 } & Number & $22(49 \%)$ & $17(62.6 \%)$ \\
\hline \multirow{2}{*}{ Stage } & $<4$ & $23(51 \%)$ & $10(37 \%)$ \\
\cline { 2 - 4 } & 4 & 21 & 5 \\
\cline { 2 - 4 } & Number & $14(66.7 \%)$ & $3(60 \%)$ \\
\cline { 2 - 4 } & Mutated & $7(33.3 \%)$ & $2(40 \%)$ \\
\hline
\end{tabular}

Citation: Ahmad S Khalil, Kamal H Al Zahran, Evelyne G Helou, Antoine A Abboud. Right Versus Left-Sided Colon Cancer: Analysis of Epidemiology in Lebanese Patients. Res \& Rev Health Care Open Acc J 3(4)- 2019. RRHOAJ.MS.ID.000169. D0I: 10.32474. RRHOAJ.2019.03.000169. 
Table 2: Epidemiologic differences between the 2-age group.

\begin{tabular}{|c|c|c|c|}
\hline \multicolumn{2}{|c|}{ Patients } & $<50$ years & $\geq 50$ years \\
\hline \multicolumn{2}{|c|}{ Number } & $10(13.9 \%)$ & $62(86.1 \%)$ \\
\hline \multicolumn{2}{|c|}{ Male } & $4(40 \%)$ & $37(59.7 \%)$ \\
\hline \multicolumn{2}{|c|}{ Female } & $6(60 \%)$ & $25(40.3 \%)$ \\
\hline \multicolumn{2}{|c|}{ Right-sided } & $2(20 \%)$ & $25(40.3 \%)$ \\
\hline \multicolumn{2}{|c|}{ Left-sided } & $8(80 \%)$ & $37(59.7 \%)$ \\
\hline \multicolumn{2}{|c|}{ Stage 4} & $4(40 \%)$ & $29(46.8 \%)$ \\
\hline \multicolumn{2}{|c|}{ < stage 4} & $6(60 \%)$ & $33(53.2 \%)$ \\
\hline \multirow{2}{*}{ RAS } & Mutated & $3(75 \%)$ & $14(64 \%)$ \\
\hline & Wild & $1(25 \%)$ & $8(36 \%)$ \\
\hline
\end{tabular}

With respect to patients with right-sided colon cancer, 2 patients had stage 4 tumor with wild type RAS, both treated with EGFR targeted therapy. One patient passed away after progression in his disease status and the other one didn't respond to first line treatment.

\section{Discussion}

Patients with RCC were, overall, older, more often of female gender, and had more advanced AJCC stages than patients with LCC [1]. Differences in clinical presentation, epidemiology, and tumor biology between right and left-sided colon cancer have long been reported in the literature [2-6]. This study retrospectively collected the data from the archive of Geitawi University Medical Center and showed that patients with LCC were more often of female gender and had more advanced AJCC stages than patients with RCC. There were more patients $<50$ years with LCC than with RCC, and the progression of the tumor was less aggressive compared to patients $\geq$ 50 years. Our study showed that RCC is less aggressive than LCC and this result, despite the small number of patients included in our study, is in contradiction with the results published in the studies mentioned above, which may let us question the role of ethnicity and geographic factors in this issue. Thus, additional studies should be done to confirm this hypothesis. The retrospective analysis of RAS mutation status in patients with colon cancer of stage 4 showed that RAS was mutated in patients with LCC more than those with RCC. The last study of CALGB/SWOG 80405 (Alliance) showed that patients who received cetuximab and had left-sided tumor had a median overall survival (OS) of 36 months versus 16.7 months for patients with right-sided tumor. While patients who received bevacizumab had an overall survival of 31.4 months for those with left-sided tumor versus 24.2 months for those with right-sided tumor $[8,9]$. These findings played a role in changing the guidelines by limiting the use of cetuximab to patients with LCC only. Also, in our study 2 patients with RCC RAS wild type were treated with EGFR targeted therapy and both demonstrated progressive disease. These data concur with the above study and may lead us to restrict ordering RAS mutation studies to patients with RCC only, perhaps reducing the financial burden. Our study's limitations were the small number of patients included with data collected from only one center, and it didn't report overall survival or progression free survival.

\section{Conclusion}

The patients with right-sided colon cancer were more commonly males, older and had less aggressive tumor than patients with left-sided colon cancer, contrasting with the studies published internationally. Moreover, patients under the age of 50 were more often females, had LCC more than RCC, and the progression of the tumor was less aggressive than in patients above the age of 50 years.

\section{Acknowledgement}

I. Conception and design: Ahmad Saadallah Khalil, Evelyne Gerard Helou

\section{Administrative support: Antoine Abi Abboud}

III. Provision of study materials or patients: Geitawi University Medical Center

\section{Collection and assembly of data: Ahmad Saadallah Khalil}

\section{Data analysis and interpretation: All authors}

VI. Manuscript writing: All authors

VII. Final approval of manuscript: All authors

\section{References}

1. Jess P, Hansen IO, Gamborg M (2013) nationwide Danish cohort study challenging the categorisation intoright sided and left-sided colon cancer. BMJ Open.

2. Jennifer M Weiss, Patrick R Pfau, Erin S O'Connor, Jonathan King, Noelle Lo Conte, et al. (2011) Mortality by Stage for Right Versus Left Sided Colon Cancer: Analysis of Surveillance, Epidemiology and End Results Medicare Data. J Clin Oncol 29: 4401-4409.

3. Rene Warschkow, Michael C Sulz, Lukas Marti, Ignazio Tarantino, Bruno M Schmied, et al. (2016) Better survival in right-sided versus left sided stage I - III colon cancer patients. BMC Cancer 16: 554.

4. Timothy J Price, Carol Beeke, Shahid Ullah, Robert Padbury, Guy Maddern, et al. (2015) Does the Primary Site of Colorectal Cancer Impact Outcomes for Patients with Metastatic Disease? 121: 830-835.

5. Frank Benedix, Uwe Schmidt, Pawel Mroczkowski, Ingo Gastinger, HansLippert (2011) Colon carcinoma classication into right and left sided cancer or according to colonic subsite? EJSO 37(2): 134.

6. Ching Wen Huang, Hsiang Lin Tsai, Ming Yii Huang, Chun Ming Huang, Yung Sung Yeh (2015) et al Different clinicopathologic features and favorable outcomes of patients with stage III left sided colon cancer. World Journal of Surgical Oncology (13): 257.

7. J C von Einem, V Heinemann, L Fischer von, Weikersthal, U Vehling Kaiser, et al. Left sided primary tumors are associated with favorable prognosis in patients with KRAS codon 12/13 wild-type metastatic colorectal cancer treated with cetuximab plus chemotherapy. Cancer Res Clin Oncol 140: 1607-1614.

8. Sabine Tejpar, Sebastian Stintzing, Fortunato Ciardiello, Josep Tabernero, Eric Van Cutsem, Frank et al. (2016) Prognostic and Predictive Relevance of Primary Tumor Location in Patients With RAS Wild Type Metastatic Colorectal Cancer. JAMA Oncol 3(2): 194-201.

9. Alan P Venook, Donna Niedzwiecki, Federico Innocenti, Briant Fruth, Claire Greene, et al. (2016) Impact of primary (1을 tumor location on overall survival (OS) and progression free survival (PFS) in patients (pts) with metastatic colorectal cancer (mCRC): Analysis of CALGB/ SWOG 80405 (Alliance). J Clin Oncol 34 (suppl; abstr 3504).(Alliance). J Clin Oncol 34 (suppl; abstr 3504). 
(C) (i) This work is licensed under Creative To Submit Your Article Click Here:

DOI: $10.32474 /$ RRHOAJ.2019.03.000169

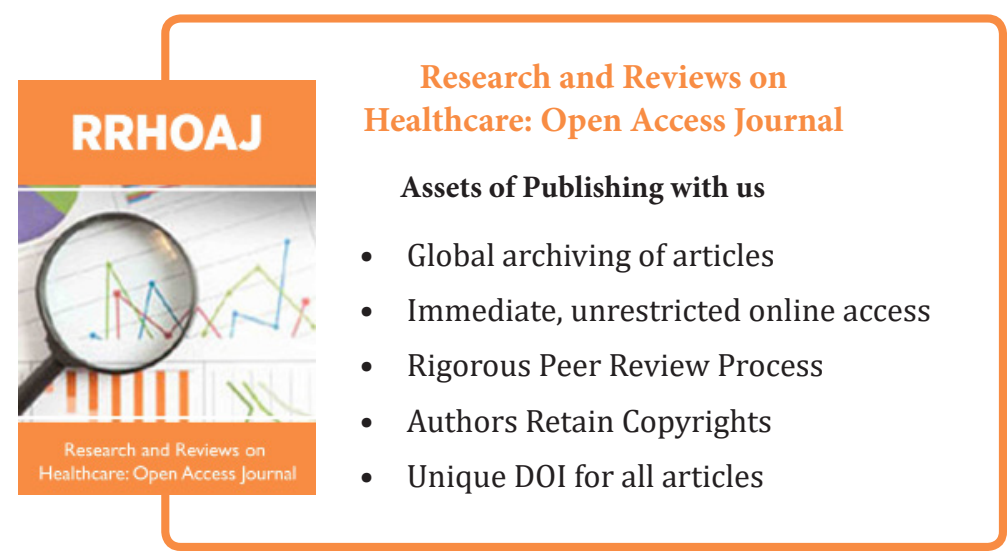

